www.nature.com/pj

\title{
Microgel-like network polymer precursor formation in free-radical cross-linking multiallyl polymerization
}

\author{
Hiroyuki Hamamoto, Kota Himei, Satoshi Inoue, Hiroyuki Aota and Akira Matsumoto
}

The present study is an extension of our preceding work on gelation behavior. We observed no substantial difference in the actual gel points among three isomeric diallyl phthalates: diallyl phthalate, diallyl isophthalate and diallyl terephthalate. The resulting network polymer precursors (NPPs) were characterized by size-exclusion chromatography with both multiangle laser light scattering and viscometry. It is of note that the structure of NPP, consisting of oligomeric primary polymer chains, becomes core-shell type dendritic or nanogel-like with the progress of polymerization. The nanogel-like NPPs can then collide with one another to form cross-links, eventually leading to gelation. Although the concentration of NPP should be high at the conversion close to the gel point, the dilution of NPP by adding monomer could prevent the cross-link formation among NPPs, and consequently lead to the successive growth of high-molecular-weight NPP from a nanogel to a microgel. The further growth of the microgel as an inhomogeneous NPP with high cross-link density could eventually reach an extremely inhomogeneous network polymer. These processes were pursued as typical examples using the bulk polymerization of DAT.

Polymer Journal (2010) 42, 923-927; doi:10.1038/pj.2010.96; published online 27 October 2010

Keywords: cross-linking; gelation; microgel; multiallyl polymerization; nanogel; network polymer precursor

In our previous work, ${ }^{1}$ the gel points in the free-radical polymerizations of diallyl aromatic dicarboxylates-including three isomeric diallyl phthalates: diallyl phthalate (DAP), diallyl isophthalate (DAI) and diallyl terephthalate (DAT) - were experimentally reexamined in detail and discussed according to Gordon's theory. ${ }^{2}$ Although the discrepancy between actual and theoretical gel-point conversions was quite large and, moreover, it was enhanced in the order DAP $<$ DAI $<$ DAT, no substantial difference in the actual gel points was observed between the three isomeric DAPs. This interesting gelation behavior was discussed in detail ${ }^{3}$ in terms of the correlation between gelation and the difference in cyclization modes, ${ }^{4}$ as well as the difference in reactivity between the uncyclized and cyclized radicals for cross-linking. Conclusively, the nonconsecutive addition in DAT polymerization led to a delayed gelation, and the cyclized radical in DAP polymerization showed an enhanced reactivity for cross-linking.

Then, we tried to extend our previous discussion ${ }^{3}$ to the polymerization of triallyl trimellitate (TAT) because the chemical structure of TAT essentially exhibits the characteristics of three isomeric DAPs (Figure 1). Therefore, the enhanced gelation was expected in TAT polymerization, involving both abundant unreacted pendant double bonds and cyclized radicals as two significant factors responsible for an enhanced intermolecular cross-linking. However, no promoted gelation was observed in TAT polymerization when compared with DAP, DAI and DAT polymerizations.
For a full understanding of the gelation behavior in cross-linking multiallyl polymerization, our preceding work $^{5}$ was focused on the characterization of resulting NPPs using size-exclusion chromatography (SEC) with both multiangle laser light scattering (MALLS) and viscosity detectors. SEC-MALLS-viscometry is a multi-detection system providing the correlation of intrinsic viscosities $([\eta])$ versus weight-average molecular weights $\left(M_{\mathrm{w}}\right)$ of fractionated samples; thus, it is a powerful tool for the characterization of NPPs with core-shell type dendritic structures. The structures of the resulting NPPs were also characterized by both correlations of [ $\eta]$ versus $M_{\mathrm{w}}$ and the r.m.s. radius of gyration versus $M_{\mathrm{w}}$. It is of note that the structure of NPP, which consists of oligomeric primary polymer chains, as a characteristic of allyl polymerization, ${ }^{6}$ would become core-shell type dendritic or nanogel-like with the progress of polymerization. This kind of nanogel-like NPP formation is inevitably related to the fact that no substantial difference in the actual gel points among DAP, DAI, DAT and TAT polymerizations was observed.

As an extension of our preceding work, ${ }^{5}$ the present study further pursued the highest-molecular-weight DAT-NPP, that is, the nanogellike NPP. It should be recalled that we have been concerned with crosslinking multiallyl polymerization ${ }^{7,8}$ as compared with that of the opposite case of multivinyl systems, in which numerous reports about microgel formation were published on the homopolymerization of common multivinyl monomers and their copolymerization with common monovinyl monomers. ${ }^{9-13}$ In our previous investigation, ${ }^{14}$

Department of Chemistry and Materials Engineering, Faculty of Chemistry, Materials and Bioengineering \& High Technology Research Center, Kansai University, Suita-shi, Osaka, Japan

Correspondence: Professor A Matsumoto, Department of Chemistry and Materials Engineering, Faculty of Chemistry, Materials and Bioengineering \& High Technology Research Center, Kansai University, 3-3-35 Yamate-cho, Suita-shi, Osaka 564-8680, Japan.

E-mail: amatsu@kansai-u.ac.jp

Received 22 July 2010; revised 5 September 2010; accepted 7 September 2010; published online 27 October 2010 
<smiles>C=CCOC(=O)c1ccccc1C(=O)OCC</smiles>

DAP<smiles>C=CCOC(=O)c1cccc(C(=O)OCC)c1</smiles>

DAI<smiles>C=CCOC(=O)c1ccc(C(=O)OCC)cc1</smiles>

DAT<smiles>C=CCOC(=O)c1ccc(C(=O)OCC)c(C(=O)OCC=C)c1</smiles>

TAT

Figure 1 Chemical structures of DAP, DAI, DAT and TAT.

we tried to clarify the inhomogeneous cross-link structure formation or the microgelation in the bulk polymerization of DAP because the cross-linked DAP resin consisted of colloidal particles when observed using electron microscopy. ${ }^{15}$ However, our results clearly demonstrated that any microgelation leading to the formation of colloidal particles did not occur before the gel point. Thereafter, more detailed work $^{5}$ using SEC-MALLS-viscometry revealed nanogel formation in place of microgelation, as mentioned above.

Through this connection, the most significant difference between allyl and common vinyl polymerizations is in the predominant influence of the length of the primary polymer chain on gelation. ${ }^{2,16,17}$ In diallyl polymerization, only the oligomeric primary polymer chain is formed because the occurrence of a monomer chain transfer is quite remarkable. ${ }^{5}$ Thus, the structure of the resulting NPPs in cross-linking multiallyl polymerization changed with conversion and eventually became nanogel-like at a conversion close to the gel point. Then, the nanogel-like NPPs were able to collide with each other to form cross-links with other NPPs, eventually leading to macrogel formation or gelation. Although the concentration of the NPP should be high at a conversion close to the gel point, the dilution of NPP by newly added monomer could prevent the cross-link formation among NPPs, and consequently lead to the growth of NPP from a nanogel to a microgel. The further growth of this type of microgel as an inhomogeneous NPP with high cross-link density could eventually result in a macrogel of an inhomogeneous giant molecule. This macrogel will provide a typical example of an extremely inhomogeneous network polymer formation. Thus, we explored the successive growth of a highest-molecular-weight NPP with high cross-link density to a microgel by means of nanogel formation in free-radical cross-linking multiallyl polymerization.

\section{EXPERIMENTAL PROCEDURE}

\section{Materials}

DAT (Daiso, Osaka, Japan) as a monomer was purified by vacuum distillation under nitrogen. Benzoyl peroxide (NOF, Aichi, Japan) as an initiator was purified by a conventional method.

\section{Polymerization}

Polymerization was carried out in a glass ampule containing suitable amounts of monomer and initiator. The ampule was degassed three times by the usual freezing and thawing technique under vacuum and then sealed. It was then placed in a thermostat regulated at $80^{\circ} \mathrm{C}$. After a predetermined reaction time, the polymer was precipitated by pouring the reaction mixture into a large excess of hexane containing a small amount of 4-tert-butylpyrocatechol as an inhibitor. The purification of the polymer was done by reprecipitation from a tetrahydrofuran-precipitant system. The gel fraction of the polymer at a conversion beyond the gel point was separated by extracting the sol fraction with tetrahydrofuran.

\section{Measurements}

The $M_{\mathrm{w}}$ of the resulting NPPs were measured by light scattering. The light scattering measurements were carried out in tetrahydrofuran at $30^{\circ} \mathrm{C}$ with an
Otsuka Electronics DLS-7000 dynamic light scattering spectrophotometer over the angular range between 30 and $150^{\circ} \mathrm{C}$ using unpolarized light at a wavelength of $632.8 \mathrm{~nm}$. The resulting NPPs were also subjected to SECMALLS-viscosity measurement in order to estimate the correlation of $[\eta]$ versus $M_{\mathrm{w}}$ by SEC using a triple detector system set in the direction of flow. This system consisted of a viscometer, MALLS device and a differential refractometer in sequence. The SEC-MALLS-viscosity measurements were carried out at $40^{\circ} \mathrm{C}$ in tetrahydrofuran using a three-column Shodex GPC KF-806 L at polymer concentrations of $0.1-0.5 \%(\mathrm{w} / \mathrm{v})$ and a flow rate of $1 \mathrm{ml} \mathrm{min}{ }^{-1}$. The MALLS device was a DAWN model F (Wyatt Technology, Santa Barbara, CA, USA); the laser beam used a wavelength of $632.8 \mathrm{~nm}$ and a $67-\mu$ l flow cell. A Wyatt ViscoStar (Wyatt Technology) was employed as the viscometer.

\section{RESULTS AND DISCUSSION}

Characteristics of Dendritic NPPs Consisting of Oligomeric Primary Polymer Chains as Exemplified by DAT Polymerization Here, it is worthy to note that allyl monomers polymerize only with difficulty, and their polymerization yields polymers having low $M_{\mathrm{w}}$, that is, oligomers. ${ }^{18-21}$ Inevitably, free-radical cross-linking multiallyl polymerization provides network polymers consisting of oligomeric primary polymer chains. ${ }^{7,8}$ At an early stage of polymerization, only oligomeric linear or loop-structured NPP is formed to generate the core of the network polymer, which is completely opposed to the case of common multivinyl polymerization in which a swollen highmolecular-weight polymer particle is presumed to be formed. ${ }^{22,23}$ With the progress of polymerization, an enhanced occurrence of intermolecular cross-linking enlarges the core from a linear polymer to a branched one. This branched NPP grows further to give a highly branched NPP, and eventually gelation occurs as a result of indefinitely large network polymer formation. It is of note that the structure of NPP consisting of oligomeric primary polymer chains becomes core-shell type dendritic with the progress of polymerization as is typically exemplified by the monoallyl/diallyl copolymerization shown in Figure 2. This kind of core-shell type dendritic NPP formation as a characteristic of the highly branched NPP consisting of oligomeric primary polymer chains generated from allyl polymerization may be related to the fact that no substantial difference in the actual gel points among DAP, DAI, DAT and TAT polymerizations was observed. This relationship may fall into the category of Flory-Stockmayer gelation theory or a tree model. ${ }^{16,24,25}$

Thus, the polymerization of DAT was conducted in bulk using $0.1 \mathrm{moll}^{-1}$ benzoyl peroxide at $80^{\circ} \mathrm{C}$. Figure 3 shows the conversion versus time curve, as well as the percentage of gel polymer. It is of note that no gel effect ${ }^{26}$ was observed, even beyond the gel point. The time at which a gel starts to form was then determined by extrapolating the gel formation curve to a zero percentage. The conversion at which gel starts to form, that is, the gel point, was estimated to be $25.8 \%$, although the gel points in DAP and TAT polymerization were estimated to be 26.8 and $25.3 \%$, respectively. Figure 4 shows the SEC curves of NPPs obtained using DAT polymerization after $40,60,80$ and $90 \mathrm{~min}$. The molecular-weight 


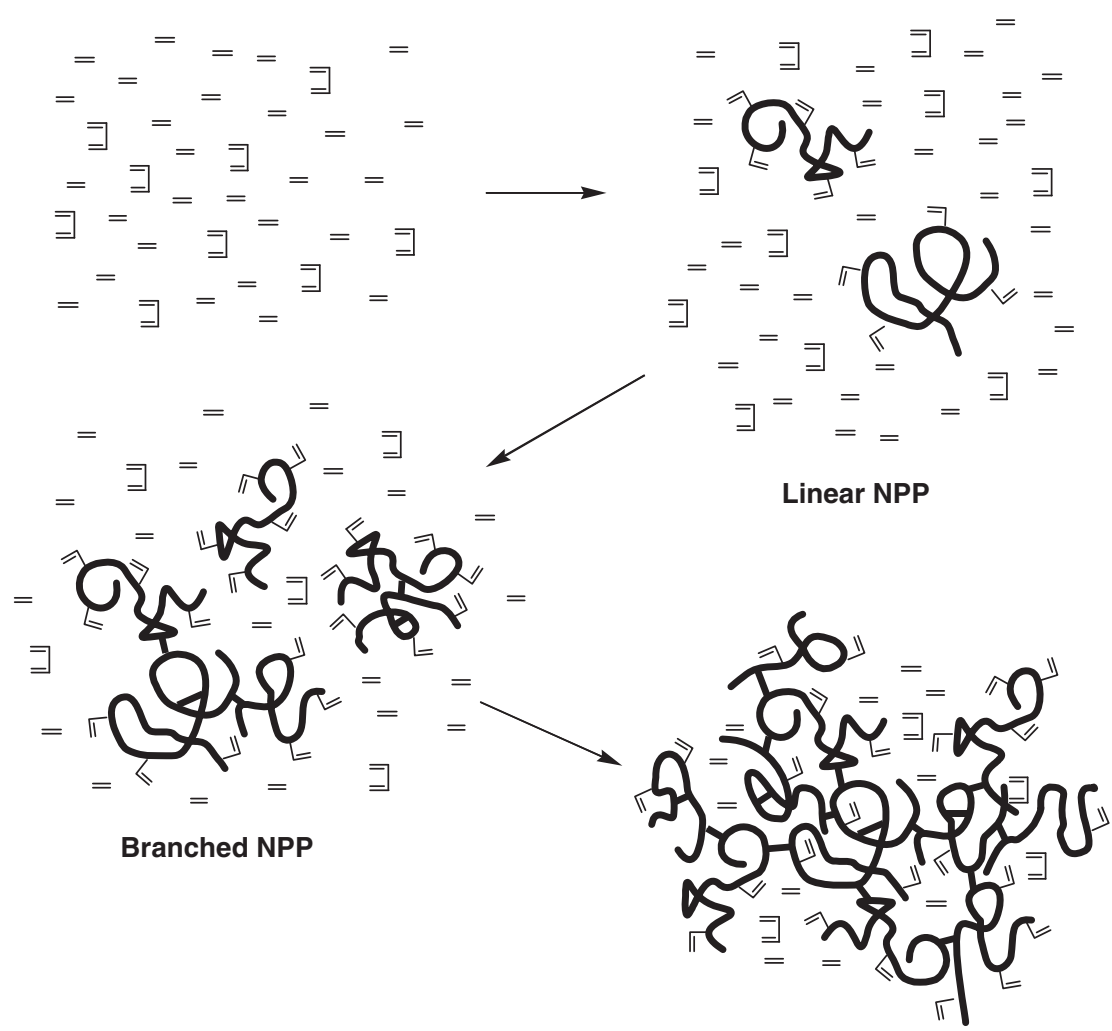

Core-shell type dendritic NPP

Figure 2 A rough sketch of network formation processes in the free-radical cross-linking monoallyl/diallyl copolymerization.

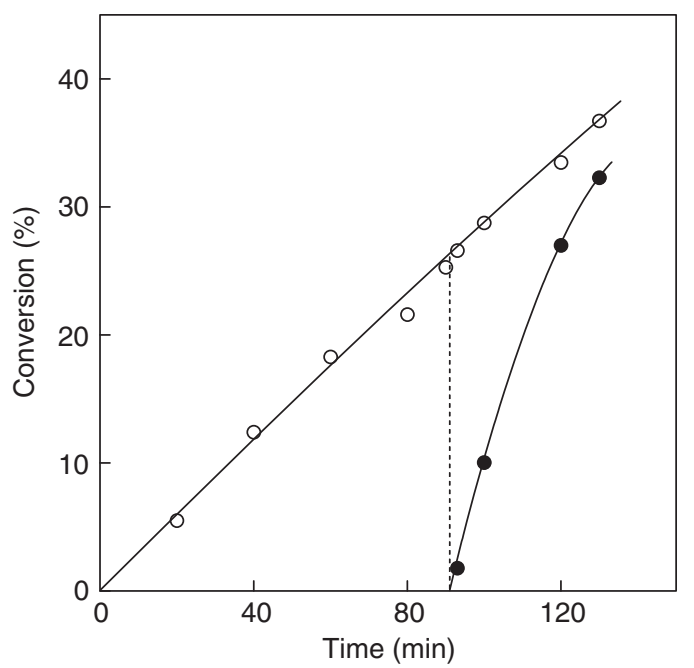

Figure 3 Conversion versus time curve for the bulk polymerization of DAT using $0.1 \mathrm{moll}^{-1}$ benzoyl peroxide at $80^{\circ} \mathrm{C}$. Open and filled symbols represent total and gel polymers, respectively.

distributions of the resulting DAT-NPPs were broadened toward a higher-molecular-weight side with the progress of polymerization as a reflection of intermolecular cross-linking. It is of note that, up to the polymerization at $80 \mathrm{~min}$, the broadening tendency of molecular-weight distribution was gradual, and then it became steep as the conversion approached the gel point. The latter steep

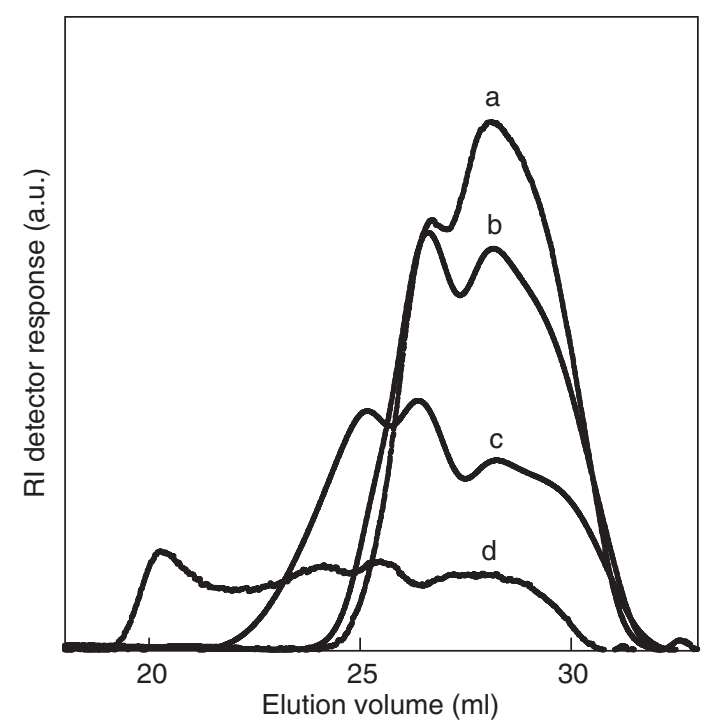

Figure 4 RI-monitored SEC curves of NPPs obtained in DAT polymerization for (a) 40, (b) 60, (c) 80 and (d) 90 min (see Figure 3).

broadening tendency of molecular-weight distribution supports our expectation that the core-shell type dendritic or nanogel-like NPPs with a nanosolid as the core part can collide with each other to form cross-links with other NPPs. This cross-linking eventually leads to gelation as a reflection of the high concentration of NPP at a conversion close to the gel point. This was also shown in Figure 5 


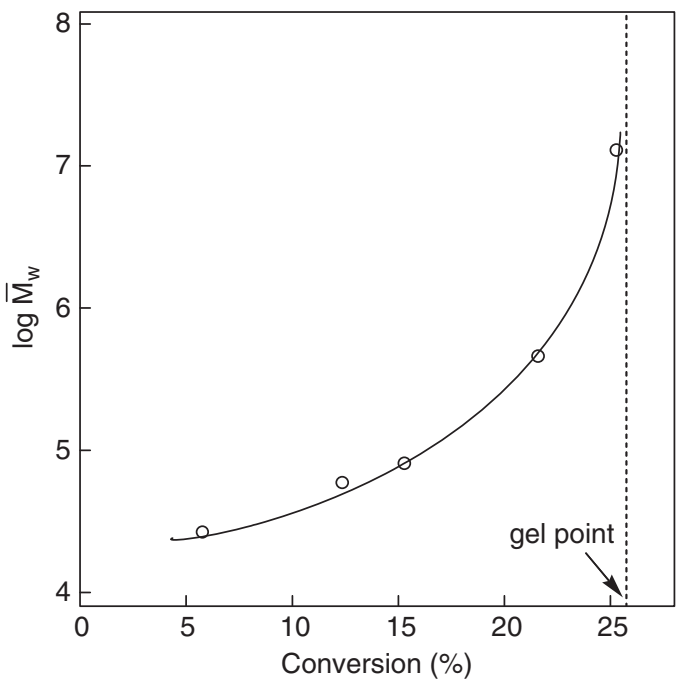

Figure 5 Dependence of $M_{\mathrm{w}}$ on conversion (see Figure 3).

as the dependence of $M_{\mathrm{w}}$ of the resulting NPP on conversion; the increasing tendency of $M_{\mathrm{w}}$ with conversion became remarkable at a conversion close to the gel point in which the fluidity of the polymerization solution was completely lost.

\section{Successive growth of highest-molecular-weight NPP with high cross-link density to microgel by nanogel formation}

As mentioned above, the nanogel-like NPPs could collide with each other to form cross-links among NPPs as a reflection of the high concentration of NPP at a conversion close to the gel point. Accordingly, the dilution of NPP by newly added monomer would induce the inhibition of cross-link formation between NPPs. Consequently, it may lead to the successive growth of highest-molecular-weight NPP with high cross-link density to microgel by nanogel formation. Thus, we attempted to conduct the stepwise addition polymerization as follows: (a) DAT was polymerized in bulk using $0.1 \mathrm{moll}^{-1}$ benzoyl peroxide at $80^{\circ} \mathrm{C}$ for $40 \mathrm{~min}$, (b) the polymerization solution was diluted to one-half by adding the same volume of monomer containing $0.1 \mathrm{moll}^{-1}$ benzoyl peroxide and the polymerization was continued for $20 \mathrm{~min}$, (c) the polymerization solution was diluted similarly and, the polymerization was continued again for $20 \mathrm{~min}$ and (d) repeated 11 times.

Figure 6 shows the SEC curves of DAT-NPPs obtained in the stepwise-addition polymerization after 40, 60, 80 and $90 \mathrm{~min}$. The broadening tendency of molecular-weight distribution to a higher molecular weight with the progress of polymerization was stepwise, which is completely different from the results shown in Figure 4 . This kind of stepwise growth of the nanogel was reflected as a rather linear plot of $M_{\mathrm{w}}$ versus time in Figure 7, being completely opposed to the steep increasing tendency of $M_{\mathrm{w}}$ as a dotted line for comparison. It is of note that the polymerization solution became turbid after the stepwise polymerization at $180 \mathrm{~min}$. Although in the bulk polymerization of DAP, ${ }^{14}$ no microgelation occurred before the gel point, as shown by the observation that no turbidity of the polymerization solution was observed. Thus, we succeeded in increasing the particle size from a nanogel to a microgel by means of our stepwise-addition polymerization method. This was supported by the quite low [ $\eta]$ value; thus, the correlation of $[\eta]$ versus $M_{\mathrm{w}}$ is plotted in Figure 8. Here, it should be noted that the intrinsic viscosity ratio, $[\eta]_{\mathrm{NPP}} /$

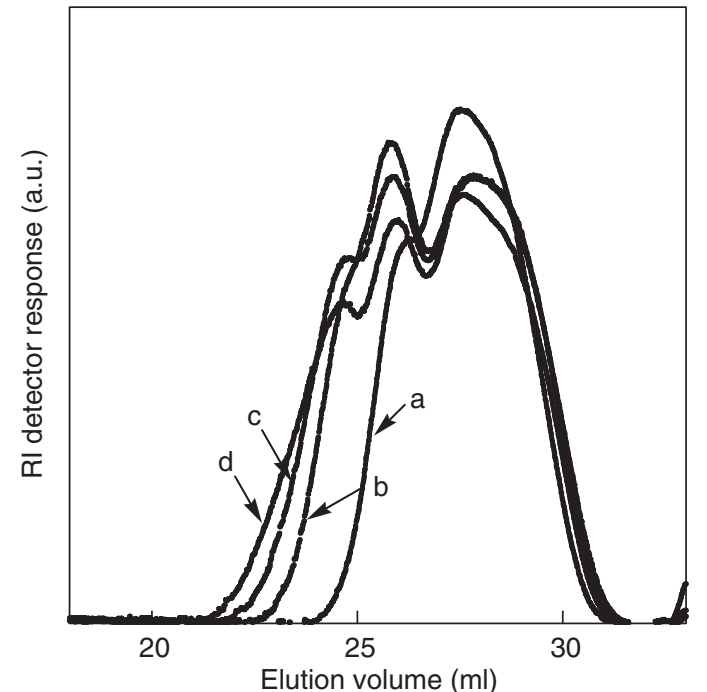

Figure 6 RI-monitored SEC curves of NPPs obtained in the stepwise addition polymerization of DAT for (a) 40, (b) 60, (c) 80 and (d) $90 \mathrm{~min}$.

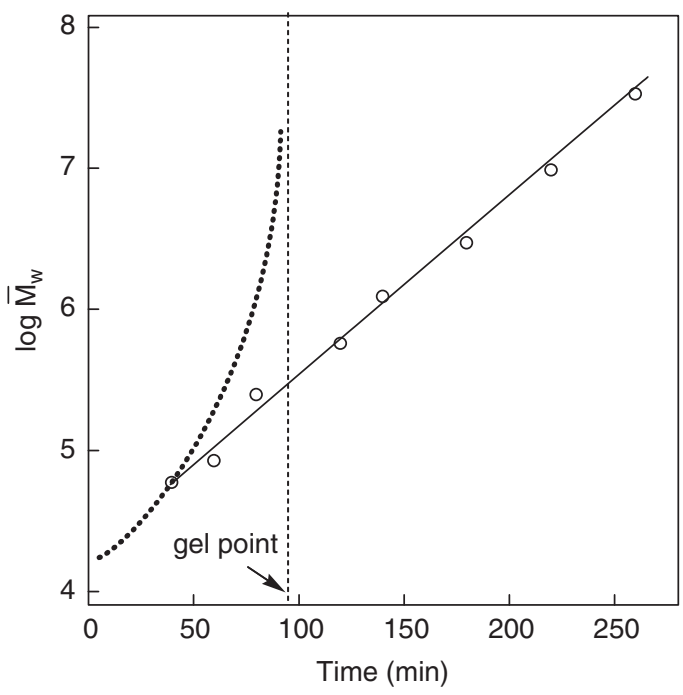

Figure 7 Dependence of $M_{\mathrm{w}}$ on time for the stepwise addition polymerization of DAT. Dotted line corresponds to the bulk polymerization of DAT (see Figure 5).

$[\eta]_{\text {Linear }}$, is well known as a measure of branching. ${ }^{27}$ Therefore, the $[\eta]$ values of NPPs were divided by those of the corresponding linear PSt with the same $M_{\mathrm{w}}$. Then, the ratios were plotted against $M_{\mathrm{w}}$, as shown in Figure 9. Quite low values were observed, suggesting the nanogel- and microgel-like DAT-NPP formation is in conformity with our expectations. Moreovre, it is of note that after $260 \mathrm{~min}$, the polymerization solution became white, supporting the microgel-like DAT-NPP formation.

\section{CONCLUSIONS}

In our previously published article, ${ }^{5}$ we explored the polymerization of DAP, DAT and TAT, especially focusing on the characterization of the resulting NPPs using SEC-MALLS-viscometry. It is of note that the structure of NPP consisting of oligomeric primary polymer chains became core-shell type dendritic or nanogel-like during the progress 


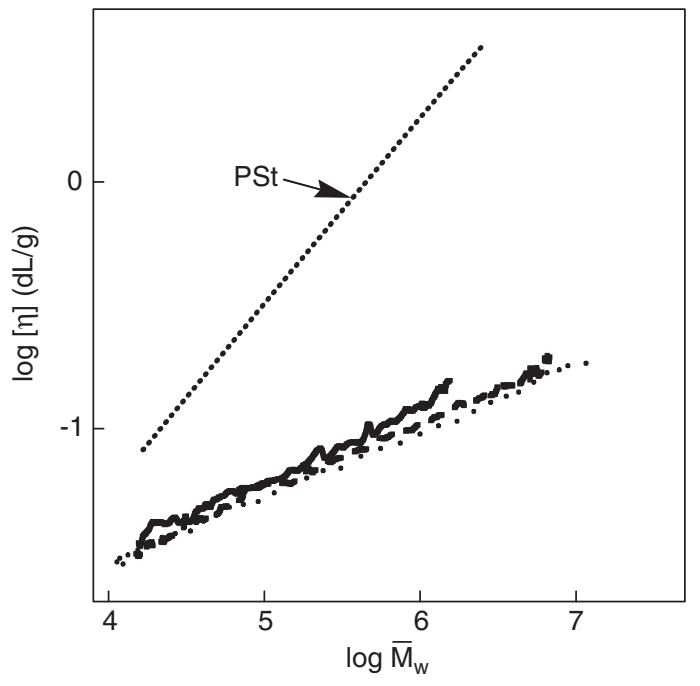

Figure 8 Double logarithmic plots of $[\eta]$ versus $M_{\mathrm{w}}$ (see Figure 7), along with a plot for polystyrene (PSt): (solid line) $60 \mathrm{~min}$, (broken line) $100 \mathrm{~min}$ and (dotted line) $140 \mathrm{~min}$.

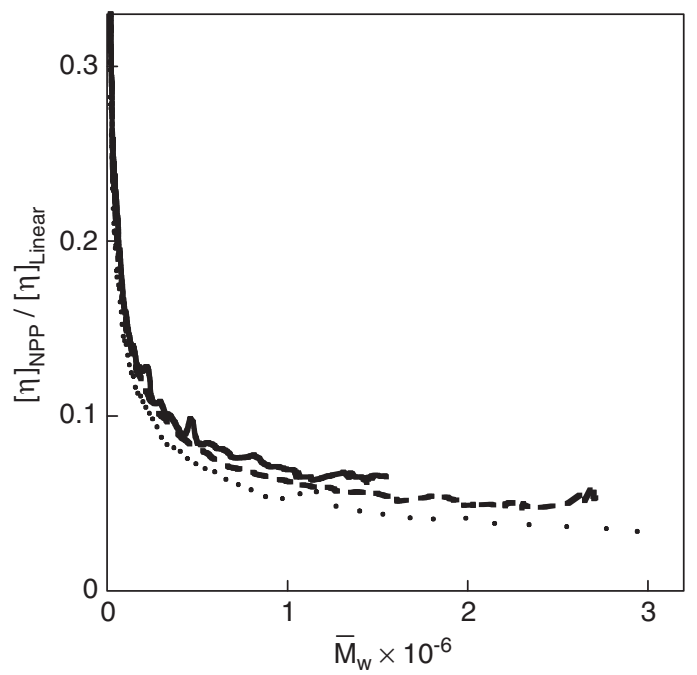

Figure 9 Plots of $[\eta]_{\mathrm{NPP}} /[\eta]_{\text {Linear }}$ versus $M_{\mathrm{w}}$ (see Figure 7 ).

of polymerization. Then the nanogel-like NPPs with a nanosolid as the core part are able to collide with each other to form cross-links, eventually leading to gelation. Although the concentration of NPP should be high at a conversion close to the gel point, the dilution of NPP by newly adding monomer could prevent the cross-link formation between NPPs, and consequently lead to the growth of NPP from a nanogel to a microgel. This possibility prompted us to attempt the successive growth of highest-molecular-weight NPP with high cross-link density from a nanogel to a microgel in a free-radical cross-linking multiallyl polymerization; before gelation, the polymerization solution eventually became quite white, supporting the microgel-like DAT-NPP formation.

\section{ACKNOWLEDGEMENTS}

This research was financially supported by the 'High-Tech Research Center' Project for Private Universities: a matching fund subsidy from the Ministry of Education, Culture, Sports, Science and Technology, 2005-2009.

1 Matsumoto, A., Yokoyama, S., Khono, T. \& Oiwa, M. Studies of the polymerization of diallyl compounds. XXV. Gel point in the polymerization of diallyl esters of aromatic dicarboxylic acids. J. Polym. Sci. Polym. Phys. Ed. 15, 127-136 (1977).

2 Gordon, M. Network theory of the gel point and the 'incestuous' polymerization of diallyl phthalate. J. Chem. Phys. 22, 610-613 (1954).

3 Matsumoto, A., Ogasawara, Y., Nishikawa, S., Aso, T. \& Oiwa, M. Studies of the polymerization of diallyl compounds. XLI. Discussion of substantially identical gel points among three isomeric diallyl phthalates. J. Polym. Sci.: Part A: Polym. Chem. 27, 839-845 (1989).

4 Matsumoto, A., Sasaki, H. \& Oiwa, M. Polymerization of diallyl compounds. XVI. Copolymerizations of diallyl esters of aromatic dicarboxylic acids with methylallyl benzoate. Makromol. Chem. 166, 179-188 (1973).

5 Matsumoto, A., Hamamoto, H., Miwa, Y., Aota, H., Inoue, S., Yokoyama, K., Matoba, Y \& Shibano, M. Discussion of substantially identical gel points among multiallyl monomers based on characterization of resultant network polymer precursors consisting of oligomeric primary polymer chains. J. Polym. Sci.: Part A: Polym. Chem. 47, 2871-2881 (2009).

6 Altschul, P. D. Polymerization of allyl compounds. II. Preliminary kinetic study of the peroxide-induced polymerization of allyl acetate. J. Am. Chem. Soc. 67, 816-822 (1945).

7 Matsumoto, A. Free-radical crosslinking polymerization and copolymerization of multivinyl compounds. Adv. Polym. Sci. 123, 41-80 (1955).

8 Matsumoto, A. Polymerization of multiallyl monomers. Prog. Polym. Sci. 26, 189-257 (2001).

9 Horie, K., Otagawa, A., Muraoka, M. \& Mita, I. Calorimetric investigation of polymerization reactions. V. Crosslinked copolymerization of methyl methacrylate with ethylene dimethacrylate. J. Polym. Sci. Polym. Chem. Ed. 13, 445-454 (1975).

10 Galina, H., Dusek, K., Tuzar, Z., Bohdanecky, M. \& Sokr, J. The structure of low conversion polymers of ethylene dimethacrylate. Eur. Polym. J. 16, 1043-1046 (1980).

11 Spevacek, J. \& Dusek, K. Manifestation of microgel-like particles of styrene ethylene dimethacrylate copolymers in solution in proton and carbon-13 NMR spectra. J. Polym. Sci. Polym. Phys. Ed. 18, 2027-2035 (1980).

12 Shah, A. C., Parsons, I. W. \& Haward, R. N. Dilute gelling systems: copolymers of styrene and glycol dimethacrylates. Polymer 21, 825-828 (1980).

13 Leicht, R. \& Furrmann, J. Network formation during styrene-divinylbenzene copolymerization investigated by the fluorescence polarization method. Polym. Bull. 4, 141-148 (1981).

14 Matsumoto, A., Nakajima, H. \& Oiwa, M. Polymerization of diallyl compounds. XLIV. Three-dimensional network formation of diallyl phthalate: microheterogenization of gel during polymerization beyond gel point. Netsukokasei. Jushi. (J. Thermoset. Plast. Jpn.) 9, 141-148 (1988).

15 Erath, E. H. \& Robinson, M. Colloidal particles in thermosetting resins. J. Polym. Sci. C3, 65-76 (1963)

16 Stockmayer, W. H. Theory of molecular size distribution and gel formation in branchedchain polymers. J. Chem. Phys. 11, 45-55 (1943).

17 Stockmayer, W. H. Theory of molecular size distribution and gel formation in branched polymers. II. General cross-linking. J. Chem. Phys. 12, 125-131 (1944).

18 Odian, G. Principles of Polymerization, 4th edn, 263-264 (Wiley, New York, 2004).

19 Schildknecht, C. E. Allyl Compounds and Their Polymers (Wiley, New York, 1973).

20 Laible, R. C. Allyl polymerizations. Chem. Rev. 58, 807-843 (1958).

21 Volodina, V. I., Tarasov, A. I. \& Spasskii, S. Polymerization of allyl compounds. Russ. Chem. Rev. 39, 140-155 (1970).

22 Walling, C. Gel formation in addition polymerization. J. Am. Chem. Soc. 67, 441-447 (1945).

23 Dusek, K. \& Duskova-Smrckova, M. Network structure formation during crosslinking of organic coating systems. Prog. Polym. Sci. 25, 1215-1260 (2000).

24 Flory, P. J. Molecular size distribution in three-dimensional polymers. I. Gelation. J. Am. Chem. Soc. 63, 3083-3090 (1941).

25 Matsumoto, A., Yokoyama, S., Khono, T. \& Oiwa, M. Studies of the polymerization of diallyl compounds. XXV. Gel point in the polymerization of diallyl esters of aromatic dicarboxylic acids. J. Polym. Sci. Polym. Phys. Ed. 15, 127-136 (1977).

26 Trommsdorff, E., Kohle, H. \& Lagally, P. Polymerization of methyl methacrylate. Makromol. Chem. 1, 169-198 (1948).

27 Zimm, B. H. \& Stockmayer, W. H. The dimensions of chain molecules containing branches and rings. J. Chem. Phys. 17, 1301-1314 (1949). 